\title{
Aprendizagem de sequências numéricas: pesquisa sobre dificuldades de Licenciandos em Matemática
}

\section{Learning about numerical sequences: a research on mathematics undergraduate difficulties}

\author{
Eleni Bisognin ${ }^{1}$ \\ Vanilde Bisognin ${ }^{2}$ \\ José Carlos Pinto Leivas ${ }^{3}$
}

\begin{abstract}
Resumo
Neste artigo, apresenta-se relato parcial de uma pesquisa desenvolvida com 15 alunos de cursos de Licenciatura em Matemática de duas Instituições de Ensino Superior do Rio Grande do Sul, Brasil. O objetivo desta investigação é analisar dificuldades encontradas por licenciandos de Matemática ao resolver uma questão sobre sequências. Como instrumento de pesquisa, foi empregado um teste composto por uma questão sobre sequência numérica, cujas respostas foram corrigidas usando as seguintes categorias: corretas, parcialmente corretas, incorretas e em branco. A análise foi feita por meio de descrição e comentários das respostas, com base em teorizações sobre os Três Mundos da Matemática. De maneira geral, constata-se que os licenciandos participantes da pesquisa apresentam dificuldades, tanto conceituais como procedimentais, relacionadas ao conceito de limite de uma sequência e parecem estar, ainda, nos estágios da matemática prática e teórica, sem atingir o estágio da matemática formal.
\end{abstract}

Palavras-chave: Sequências numéricas; Licenciandos em Matemática; Análise de respostas.

\begin{abstract}
In this article, we present partial report of a survey conducted with 15 students of Mathematics Teacher Training courses at two higher education institutions of Rio Grande do Sul, Brazil. The purpose of this research is to analyze difficulties encountered by undergraduate mathematics to solve a question about sequences. As a research tool, it was used a test composed of a question of numerical sequence, the answers were corrected using the following categories: correct, partially correct, incorrect and no answer. The analysis was made by description and comments of responses, based on theories about the three worlds of mathematics. In general, it appears that the participants present difficulties, both conceptual and procedural, related to the concept of limit of a sequence and seems to be still in the stages of practice and theoretical mathematics, without reaching the stage of formal mathematics.
\end{abstract}

1 Doutora em Matemática pela Universidade Federal do Rio de Janeiro, Professora do Programa de PósGraduação em Ensino de Ciências e Matemática do Centro Universitário Franciscano, de Santa Maria, RS, Brasil. Email: eleni.bisognin@gmail.com

2 Doutora em Matemática pela Universidade Federal do Rio de Janeiro, Professora do Programa de PósGraduação em Ensino de Ciências e Matemática do Centro Universitário Franciscano, de Santa Maria, RS, Brasil. Email: vanildebisognin@gmail.com

${ }^{3}$ Doutor em Educação pela Universidade Federal do Paraná, Professor do Programa de Pós-Graduação em Ensino de Ciências e Matemática do Centro Universitário Franciscano, de Santa Maria, RS, Brasil. Email: leivasjc@yahoo.com.br 
Keywords: Numerical sequences; Undergraduate mathematics students; Answer analysis.

\section{Introdução}

O conhecimento do professor de Matemática não comporta apenas domínio dos conteúdos da disciplina, mas, especialmente, o conhecimento das estratégias de ensino que propiciam ao aluno a aprendizagem de determinado conteúdo. Shulman (1986, 1987) estabeleceu categorias que formam a base do conhecimento para o ensino e, entre elas, destacam-se: a) conhecimento do conteúdo; b) conhecimento pedagógico geral; c) conhecimento pedagógico do conteúdo, "aquele amálgama especial de conteúdo e pedagogia que é exclusivamente o campo dos professores, sua própria forma especial de compreensão profissional" (Shulman, 1987, p. 8). Assim, um curso de formação de professores, além das horas atualmente propostas pelas Diretrizes Curriculares Nacionais para a formação inicial em nível superior em cursos de licenciatura para disciplinas pedagógicas (Brasil, 2015), deveria, em cada disciplina de conteúdo específico, discutir as estratégias de ensino de cada tópico, em cada nível de ensino, para que o futuro professor, além do conhecimento do conteúdo e da pedagogia, possa fazer esse "amálgama" entre tais conhecimentos, conforme sugerido por Shulman.

O conteúdo de sequências numéricas, apresentado em disciplinas de Cálculo Diferencial e Integral e de Análise Matemática, pode ser abordado desde o Ensino Fundamental, quando são trabalhadas atividades para descoberta de padrões, passando pelo Ensino Médio, em que surgem nas progressões aritméticas e geométricas, até o Ensino Superior, consideradas base para o estudo de séries. Mas, como apresentar tal conteúdo de maneira que seja compreensível pelos estudantes em diferentes níveis de ensino? Quais metodologias de ensino são mais adequadas ao seu ensino? E o que os professores precisam saber sobre sequências para entender as necessidades de diferentes propostas de ensino desse conteúdo?

Moreira e Ferreira (2013, p. 1003) consideram que a "matemática do professor", nos cursos de Licenciatura em Matemática, deveria estar

[...] presente, de modo natural, em diversos lugares e momentos do currículo de formação, desde as disciplinas tradicionalmente referidas como de conteúdo matemático [...] até o Estágio Supervisionado e a Prática de Ensino, a Didática, passando, também, pelas práticas de investigação em sala de aula, modelagem matemática, resolução de problemas, pela história da matemática e da educação matemática, atravessando, inclusive, as discussões sobre avaliações e objetivos da educação escolar. (grifos do original).

Nem sempre o ensino de sequências numéricas, em especial na disciplina de Análise, aponta essa presença em possíveis abordagens nos níveis básicos de ensino; assim, julga-se que um primeiro passo para propor mudanças nas disciplinas de um curso de Licenciatura em Matemática é verificar se os alunos têm claros os conceitos envolvidos, para posteriormente 
planejarem suas aulas sobre tais tópicos para a educação básica (nos estágios ou mesmo nas suas futuras práticas). Essa verificação pode ser feita por meio de atividades em que o licenciando tenha que se expressar por escrito, para que seja possível detectar se reconhecem padrões em figuras, apresentam a sequência simbolicamente e a definem formalmente - ou seja, se apresentam características dos Três Mundos da Matemática (Tall, 2013) - e quais as dificuldades que persistem.

Os estudos sobre a produção escrita de estudantes de Matemática de qualquer nível de ensino e, em especial, sobre os erros por eles cometidos, vêm sendo desenvolvidos sob vários enfoques teóricos, de acordo com as orientações dos grupos de pesquisa nos quais o tema é discutido (Borasi, 1996; Cury, 2007; Santos, Buriasco \& Ciani, 2008; Barichello, 2008; Ramos \& Curi, 2014). É consenso que as maiores dificuldades na aprendizagem, no ensino superior da área de Ciências Exatas, são evidenciadas nas primeiras disciplinas matemáticas constantes da matriz curricular dos cursos. Nas Licenciaturas em Matemática, os problemas parecem maiores porque os estudantes, futuros professores de Matemática da educação básica, muitas vezes mostram não dominar conceitos que são ensinados no Ensino Fundamental e Médio.

A formação de professores de Matemática, inicial ou continuada, precisa levar em conta as dificuldades dos alunos em conteúdos específicos, porque imagens de conceitos (Tall \& Vinner, 1981), construídas ao longo da escolarização desses estudantes, podem conflitar com definições que lhes são apresentadas nos respectivos cursos, constituindo-se em uma fonte de erros que vão prejudicar a sua futura prática profissional.

Há várias pesquisas, realizadas no Brasil e no exterior, que enfocam o ensino de conteúdos específicos de Matemática no ensino superior, tais como equações (Attorps, 2006), cálculo diferencial e integral (Zubieta \& Meza, 2008), limites (Hardy, 2009), geometria hiperbólica (Ferreira, 2011), sistemas lineares (Nomura, 2014), entre outros, mostrando a preocupação com a reprovação e desistência de estudantes dos respectivos cursos. Entre os conteúdos basilares para a compreensão da Matemática estão as sequências numéricas, que, dependendo da matriz curricular do curso de Licenciatura em Matemática, são abordadas em diferentes disciplinas.

Considera-se que as dificuldades evidenciadas no trabalho com esses conteúdos, por alunos de cursos de Licenciatura em Matemática, têm se constituído em um problema que merece ser enfrentado por meio de uma investigação aprofundada. Dessa forma, os autores deste artigo, participantes de um projeto de pesquisa apoiado por Edital Universal do Conselho Nacional de Desenvolvimento Científico e Tecnológico $(\mathrm{CNPq})^{4}$, propuseram uma investigação a partir das seguintes questões: Quais as dificuldades apresentadas por professores de Matemática em formação inicial, em relação ao conteúdo "sequências numéricas"? Como essas dificuldades se situam em relação aos Três Mundos da Matemática,

\footnotetext{
${ }^{4}$ Processo 443118/2014-0
} 
conforme Tall $(2004,2013)$ ? Este artigo apresenta a primeira parte desta pesquisa, que tem como objetivo analisar dificuldades encontradas por licenciandos de Matemática ao resolver uma questão sobre sequências numéricas.

Uma breve revisão de livros-texto usados em cursos superiores da área de Ciências Exatas, no Brasil, mostra que a definição de sequência numérica pode ser encontrada em livros de Cálculo, de Álgebra ou de Análise, na linguagem natural ou simbólica. Malta, Pesco e Lopes (2002, p. 95) definem: “Uma sequência de números reais é uma lista de números ordenados pelos naturais, isto é, uma sequência nos dá um número real que é o primeiro termo da sequência, um número que é o $2^{\circ}$ termo da sequência e assim por diante". (grifo dos autores).

Stewart (2001, p. 693) já traz mais simbologia para a explicação: "Uma sequência pode ser pensada como uma lista de números escritos em uma ordem definida: $a_{1}, a_{2}, a_{3}, a_{4}$, $\ldots a_{n}, \ldots$ O número $a_{1}$ é chamado de primeiro termo, $a_{2}$ é o segundo termo, e em geral $a_{n}$ é o $n$ ésimo termo." (grifos do autor).

Lima (1999, p.22) define uma sequência de números reais como "uma função $x$ : $\mathbb{N} \rightarrow \mathrm{R}$, que associa a cada número natural $n$ um número real $\mathrm{x}_{\mathrm{n}}$, chamado o $n$-ésimo termo da sequência". Segundo o mesmo autor (p. 23), o número real a "é o limite da sequência $\left(x_{n}\right)$ quando, para todo número real $\varepsilon>0$, dado arbitrariamente, pode-se obter $n_{0} \in \mathbb{N}$ tal que todos os termos $x_{n}$, com índice $n>n_{0}$, cumprem a condição $\left|x_{n}-\mathrm{a}\right|<\varepsilon ”$. (grifos do autor).

Portanto, em alguma disciplina do curso de Licenciatura em Matemática, o aluno deverá ter encontrado os conceitos de sequência, limite de uma sequência, convergência de sequência e limites no infinito. Para poder trabalhar com a educação básica, é necessário que o futuro professor compreenda esses conceitos, pois vai encontrá-los em vários conteúdos do Ensino Fundamental ou Médio. Na educação básica, o tema "sequências" pode envolver, entre outros itens, o conhecimento de aproximações do número Pi e de valores de funções logarítmicas, trigonométricas ou exponenciais, bem como o próprio estudo de aproximação de funções por polinômios. Também pode ser aprofundado o estudo de progressões aritméticas e geométricas, em relação com problemas de Matemática Financeira. Dessa forma, enfocando um conteúdo que permite o trânsito entre diferentes disciplinas e níveis de ensino, é possível lidar com dificuldades de professores em formação inicial, que podem ter sido mascaradas em avaliações somativas, que investigam o desempenho final do aluno, com vistas a sua classificação ou certificação.

A fundamentação teórica para a análise dos dados desta pesquisa é baseada nas ideias de Tall $(2004,2013)$ sobre os Três Mundos da Matemática. Tais ideias são referenciadas, brevemente, a seguir.

\section{Pressupostos Teóricos}

Segundo Tall (2004, 2013), o desenvolvimento cognitivo de um sujeito, em Matemática, envolve três mundos: mundo conceitual corporificado, mundo proceitual 
simbólico e mundo formal axiomático. O mundo conceitual corporificado é relacionado à nossa percepção do mundo real e à nossa ação sobre ele. Por meio da reflexão e do uso da linguagem, podemos enfocar aspectos de nossa experiência sensorial, que nos levam a imaginar elementos provenientes da nossa visualização ou da nossa imaginação. Lima (2007, p. 74) considera que a corporificação "se refere a experiências que envolvam objetos físicos e observação, descrição, ação e reflexão sobre eles”, mas também se refere a experiências mentais, por meio das quais um indivíduo pode manipular um objeto em seu pensamento. Por exemplo, por meio da visualização de um padrão que se repete, podemos imaginar uma sequência numérica e representá-la por meio de desenhos ou de símbolos matemáticos.

O mundo proceitual simbólico é relacionado ao uso dos símbolos que utilizamos em Matemática, para indicar as ações e percepções presentes no mundo corporificado. Os símbolos representam tanto o significado dado aos conceitos quanto às ações que são efetuadas. Gray e Tall (1994) fazem uma distinção entre "processo" e "procedimento": para eles, "processo" é usado em termos gerais, como quando se menciona o processo de adição, enquanto que "procedimento" se refere a um algoritmo específico para implementar um processo, tal como o algoritmo da adição. Em seguida, os autores se referem à forma como processo e conceito são combinados em uma única noção, a que eles dão o nome de "proceito". Por exemplo, quando um matemático está usando a mesma notação para representar tanto o processo como o seu produto, tal como no símbolo 5+4, que indica, ao mesmo tempo, o processo de adição e o seu próprio resultado, a soma 9. Outro exemplo dado pelos mesmos autores envolve a notação $\lim _{n \rightarrow \infty} a_{n}$, que indica tanto o processo de tender a um limite de uma sequência quanto o conceito do valor do limite.

O mundo formal axiomático é o mundo baseado em propriedades, que são expressas em termos de definições, axiomas e teoremas que formam o sistema axiomático. As intersecções entre os mundos gerou o esquema descrito na figura 1, adaptada e traduzida de Tall (2013), no qual são detalhados esses mundos e a existência de três estágios de desenvolvimento, não hierárquicos, intitulados matemática prática, matemática teórica e matemática formal.

A matemática prática envolve experiências tais como "reconhecer e descrever figuras; dar símbolos aos elementos envolvidos; fazer cálculos aritméticos ou algébricos; enfim, atividades práticas corporificadas e simbólicas, que não envolvem características do mundo formal axiomático”. (Mação, 2014, p. 38).

O estágio da matemática teórica "inclui os níveis mais sofisticados de corporificação e simbolismo" (Tall, 2013, p. 19) e o da matemática formal refere-se ao "desenvolvimento de provas axiomáticas formais, baseadas em definições da teoria dos conjuntos e provas matemáticas de teoremas". (Ibid, p. 19). 
DOI: http://dx.doi.org/10.20396/zet.v24i3.8648090

\begin{tabular}{|c|c|c|c|}
\hline \multirow{3}{*}{$\begin{array}{l}\text { Matemática } \\
\text { Formal } \\
\text { Matemática } \\
\text { Teórica }\end{array}$} & \multicolumn{3}{|l|}{ FORMAL } \\
\hline & \multicolumn{3}{|c|}{ Formal Axiomático } \\
\hline & $\begin{array}{l}\text { Formal } \\
\text { Corporificado }\end{array}$ & $\begin{array}{l}\text { Provas } \\
\text { combinando } \\
\text { corporificação e } \\
\text { simbolismo }\end{array}$ & $\begin{array}{l}\text { Formal } \\
\text { Simbólico }\end{array}$ \\
\hline \multirow[t]{2}{*}{$\begin{array}{l}\text { Matemática } \\
\text { Prática }\end{array}$} & Espaço e Forma & $\begin{array}{l}\text { Simbólico } \\
\text { Corporificado }\end{array}$ & $\begin{array}{l}\text { Aritmética } \\
\text { generalizada } \\
\text { Aritmética } \\
\text { Números }\end{array}$ \\
\hline & & & SIMBÓLICO \\
\hline
\end{tabular}

Figura 1 - Intersecções entre os Três Mundos da Matemática

Fonte: adaptação pelos autores de figura elaborada por Tall (2013, p. 198)

Nem todos os indivíduos passam da mesma forma pelos Três Mundos da Matemática, porque há influências diversas, de seu meio ambiente, de sua formação escolar e das dificuldades que encontrou no seu aprendizado. Lima (2007) considera que, para cada conceito, os estudantes formam imagens individuais, pois, como afirmam Tall e Vinner (1981, p. 152), a imagem de conceito "é construída no decorrer dos anos por meio de experiências de todos os tipos, mudando à medida que o indivíduo encontra novos estímulos e amadurece."

Para seu desenvolvimento cognitivo, um aluno de Licenciatura em Matemática precisa passar por esses estágios e, ao fazê-lo, recorre a conhecimentos aprendidos anteriormente, que Tall intitula "met-before" e que podem ser inerentes ao assunto a ser apreendido ou frutos de uma aprendizagem anterior. Lima (2007) traduziu a expressão por "já-encontrado".

Lima e Tall (2008) consideram que um "já-encontrado" pode influenciar a aprendizagem de maneira positiva ou negativa. Um exemplo de influência positiva é o conhecimento de procedimentos para simplificar frações algébricas, que auxiliam os estudantes quando resolvem um exercício de cálculo de limite de uma função racional. No entanto, se um aluno memorizou tais procedimentos e acredita que "termos semelhantes no numerador e denominador são cancelados", ele comete erros quando "cancela" uma parcela de uma soma com a mesma parcela em outra distinta.

Sob a influência representada pelas suas experiências anteriores, licenciandos em Matemática, ainda que reconheçam suas dificuldades em termos de conteúdos ou de metodologias de ensino, muitas vezes não conseguem modificar a situação, pela falta de 
condições (de tempo, de estudos ou de orientação) para discuti-la. Analisar respostas que mostram predominância de um dos Três Mundos da Matemática, bem como dos elementos "já-encontrados" que influenciam essas respostas, é uma maneira de auxiliar esses licenciandos na superação de suas dificuldades, de forma a não gerarem obstáculos à aprendizagem de seus futuros alunos.

\section{Metodologia da Pesquisa}

Nesta pesquisa, de caráter qualitativo, foram analisadas respostas a uma questão sobre o conteúdo de sequências numéricas, aplicado a 15 alunos de Licenciatura em Matemática de duas Instituições de Ensino Superior do Estado do Rio Grande do Sul. Os alunos frequentavam os últimos semestres dos respectivos cursos, já tendo estudado o conteúdo de sequências numéricas em disciplina de Análise Matemática. A questão foi aplicada em sala de aula, tendo sido previamente solicitada aos alunos a assinatura de Termo de Consentimento Livre e Esclarecido. Cada aluno ${ }^{5}$ foi identificado pela letra A, seguida de um número, para preservar seu anonimato.

A questão é indicada a seguir:

A sequência $\left(a_{n}=\frac{n}{n^{2}+1}\right)$ tem os seus primeiros termos representados na figura abaixo.

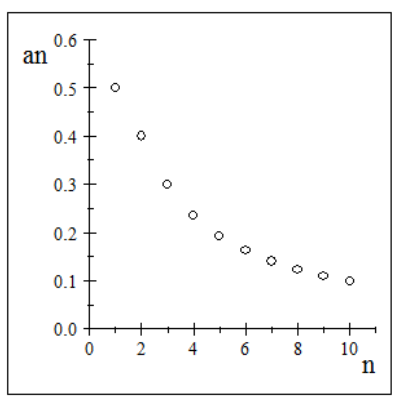

(a) A figura permite concluir que a sequência é convergente? Por quê?

(b) Considere, também, a definição do seu termo geral e determine qual é o limite desta sequência quando $n \rightarrow+\infty$.

$\mathrm{O}$ item $a$ exige conhecimento conceitual e o $b$, principalmente conhecimento procedimental. No item $a$, espera-se que o aluno justifique que a sequência converge porque, à medida que $n$ cresce, os valores da função se aproximam de zero; essa é uma resposta

\footnotetext{
${ }^{5}$ Para preservar a identidade dos(as) alunos(as) desses cursos de Licenciatura em Matemática, usou-se o gênero masculino para mencionar cada um(a) deles(as).
} 
DOI: http://dx.doi.org/10.20396/zet.v24i3.8648090

correta em linguagem natural. Mas a resposta também pode ser expressa em linguagem simbólica: a sequência $\left(a_{n}\right)$ converge para 0 porque, para cada $\varepsilon>0$, existe $n_{0} \in \mathbb{N}$ tal que $n>n_{0}$ $\Rightarrow\left|a_{n}\right|<\varepsilon$. Essas são, portanto, respostas corretas.

A resposta é parcialmente correta quando o aluno conclui que a sequência é convergente, mas usa argumentos incorretos para justificar. É incorreta quando o aluno considera que a sequência não é convergente ou não sabe decidir sobre a convergência.

No item $b$, espera-se que o aluno utilize alguma estratégia para o cálculo, obtendo $\lim _{n \rightarrow \infty} \frac{n}{n^{2}+1}=0$, em que o resultado e a estratégia corretamente apresentada caracterizam resposta correta. Considera-se que a resposta é parcialmente correta quando o aluno seleciona estratégia adequada para o cálculo do limite, mas comete erros de linguagem matemática. É incorreta quando erra o cálculo do limite ou quando simplesmente apresenta uma resposta sem qualquer desenvolvimento.

Em cada item, a resposta é considerada em branco quando o aluno não escreve qualquer expressão ou apenas copia os dados do enunciado, sem tentativa de solucionar.

\section{Apresentação e Análise dos Resultados}

Por meio da correção, foram obtidos os resultados sintetizados no Quadro 1, a seguir, em que as categorias de correção das respostas são indicadas por: C (correta), PC (parcialmente correta), I (incorreta) e EB (em branco).

Quadro 1 - Categorias de respostas por aluno

\begin{tabular}{|c|c|c|c|c|c|}
\hline \multirow{2}{*}{ Alunos } & \multicolumn{2}{|c|}{ Itens da questão } & \multirow{2}{*}{ Alunos } & \multicolumn{2}{c|}{ Itens da questão } \\
\cline { 2 - 3 } \cline { 5 - 6 } & $\mathrm{a}$ & $\mathrm{b}$ & & $\mathrm{a}$ & $\mathrm{b}$ \\
\hline $\mathrm{A} 1$ & $\mathrm{~EB}$ & $\mathrm{~EB}$ & $\mathrm{~A} 9$ & $\mathrm{I}$ & $\mathrm{I}$ \\
\hline $\mathrm{A} 2$ & $\mathrm{C}$ & $\mathrm{PC}$ & $\mathrm{A} 10$ & $\mathrm{C}$ & PC \\
\hline $\mathrm{A} 3$ & EB & PC & A11 & C & I \\
\hline A4 & I & I & A12 & PC & I \\
\hline A5 & C & PC & A13 & PC & I \\
\hline A6 & EB & EB & A14 & EB & I \\
\hline A7 & EB & I & A15 & I & I \\
\hline A8 & I & PC & \multicolumn{3}{|c}{} \\
\hline
\end{tabular}

Fonte: dados da pesquisa

Esses dados foram sintetizados no Quadro 2, com a distribuição do número de respostas em cada categoria: 
DOI: http://dx.doi.org/10.20396/zet.v24i3.8648090

Quadro 2 - Distribuição do número de respostas em cada categoria

\begin{tabular}{|c|c|c|c|c|c|c|c|c|}
\hline \multirow{2}{*}{ Item } & \multicolumn{2}{|c|}{$\mathbf{C}$} & \multicolumn{2}{|c|}{ PC } & \multicolumn{2}{|c|}{ I } & \multicolumn{2}{|c|}{ EB } \\
\cline { 2 - 9 } & $\mathbf{N .}$ & $\mathbf{\%}$ & $\mathbf{N}$. & $\mathbf{\%}$ & $\mathbf{N}$. & \% & N. & \% \\
\hline $\mathrm{a}$ & 4 & 26,7 & 2 & 13,3 & 4 & 26,7 & 5 & 33,3 \\
\hline $\mathrm{b}$ & 0 & 0,0 & 5 & 33,3 & 8 & 53,3 & 2 & 13,3 \\
\hline
\end{tabular}

Fonte: dados da pesquisa

No Quadro 2, destacam-se o fato de não haver acertos no item $b$ e a alta percentagem de respostas erradas. Como a amostra é pequena, não foram criadas categorias de erros e a análise é feita sobre todas as respostas, questão por questão, com comentários sobre as dificuldades apresentadas.

Para cada item, são apresentadas as respostas corretas, parcialmente corretas e incorretas.

\section{1) Item $a$ :}

Respostas corretas:

Os alunos A2, A5, A10 e A11 acertaram a resposta, mas apenas justificaram em linguagem natural, com algumas impropriedades nessa linguagem. A2, por exemplo, escreveu que "a sequência converge, pois quanto maior o 'n', mais próximo de zero o ponto 'á 'estará". Entretanto, an não é um ponto, é o valor da sequência, ou seja, é a ordenada do ponto $\left(\mathrm{n}, \mathrm{a}_{\mathrm{n}}\right)$. A10 escreveu: "quanto maior o número de n, mais próximo de zero a sequência se aproxima". Nesse caso, há impropriedade na linguagem matemática (número de n) e na língua natural (a sequência se aproxima mais próximo de zero?). A11 considerou que " $a$ figura permite concluir que a sequência é convergente pois ela tende para um número finito", sem identificar este número.

As respostas parecem indicar uma permanência no mundo conceitual corporificado, haja vista que se ancoram em explicações sobre proximidade e tendência, sem apresentar qualquer símbolo ou definição formal para a convergência.

Respostas parcialmente corretas:

Os alunos A12 e A13 acertaram parcialmente a resposta: A12 precisou substituir, na fórmula do termo geral, o valor de $\mathrm{n}$ por 1 e por 2 para se convencer de que "ela tem um valor determinado, se aproxima de zero", o que é não é considerada justificativa válida, pois os dois valores encontrados, $a_{1}=0,5$ e $a_{2}=0,4$, não mostram essa "aproximação" a zero. A13, da mesma forma, precisou substituir n pelos valores $2,4,6,8$ e 10, para então se convencer de que "a figura converge para zero".

Ambos, ao agir sobre o objeto matemático representado pela figura, fizeram cálculos para chegar à conclusão sobre a convergência e estão, assim, evidenciando elementos dos mundos corporificado e simbólico.

Respostas incorretas: 
DOI: http://dx.doi.org/10.20396/zet.v24i3.8648090

Os alunos A4, A8, A9 e A15 responderam incorretamente. A4 considerou que a sequência não é convergente e escreveu: "Não, pois podemos verificar a convergência da esquerda para a direita, contudo, da direita para a esquerda não é possível". Considera-se que A4 confundiu o conceito de limite no infinito com limite em um ponto, pois sua resposta parece indicar que visualizava o ponto de abscissa zero. A8 escreveu: "pela figura observamos que os valores dos termos decrescem à medida que $n$ cresce, mas não podemos concluir que seja convergente, pois não sabemos o valor assumido pela sequência". A9 apenas afirmou que "Sim". A15 afirmou que "não, pois precisamos de mais pontos para visualizar".

Essas respostas indicam uma dificuldade em generalizar as informações dadas a partir de uma figura e de uma expressão para o termo geral, pois esses alunos ficaram presos aos elementos corporificados, apoiando-se, por exemplo, na "convergência da esquerda para a direita", como afirmou A4, o que parece ser, apenas, uma visualização da tendência dos pontos. A necessidade de "visualizar mais pontos", expressa por A15, também mostra essa permanência no mundo corporificado.

\section{Item $b$ :}

Não houve respostas corretas ao item b.

Respostas parcialmente corretas:

Os alunos A2, A3, A5, A8 e A10 acertaram parcialmente a resposta ao item $b$. A2 escreveu: $\lim _{n \rightarrow \infty}=\frac{n}{n^{2}+1}$. Usou incorretamente o sinal de igualdade e não continuou, abandonando a expressão e escrevendo que: " $n<n^{2}+1$, como o denominador é maior do que o numerador, esta fração irá se aproximar de zero, mas nunca será zero". Nota-se que o aluno tem um conhecimento conceitual de limite no infinito, mas não sabe encadear os símbolos de forma a apresentar uma resposta formal. A3 escreveu: " $\lim _{n \rightarrow \infty} \frac{n}{n^{2}+1}=\frac{n}{n^{2}}=\frac{1}{n}=0$ ". Mostra saber qual procedimento usar, mas erra na linguagem matemática utilizada, visto que abandona o símbolo de limite. A5 escreveu: " $\lim _{n \rightarrow \infty} \frac{n}{n^{2}+1}=\frac{\infty}{\infty^{2}+1}=0$ ", em que repete a falta do símbolo de limite e, ainda, substitui n pelo símbolo do infinito, como se fosse um número para usar no cálculo. A8 substituiu n por x e $a_{n}$ por $\mathrm{f}(\mathrm{x})$, escrevendo, então:

$$
\text { " } \lim _{x \rightarrow \infty}=\frac{x}{x^{2}+1}=\frac{\frac{1}{x^{2}}}{\frac{x^{2}}{x^{2}}+\frac{1}{x^{2}}}=\frac{0}{1+0}=\frac{0}{1}=0 "
$$

Este aluno parece conhecer o procedimento de cálculo de um limite no infinito, mas erra detalhes da linguagem matemática. Por fim, A10 escreveu: "o limite desta sequência é zero porque quanto maiores os valores tomados para $n$, mais próximos de zero estão os resultados". Mesmo que esteja correta a ideia, solicitava-se, no item, que o respondente considerasse o termo geral e determinasse o limite. 
DOI: http://dx.doi.org/10.20396/zet.v24i3.8648090

Esses alunos mostram, portanto, que conseguem desenvolver atividades corporificadas e simbólicas, mas não conseguem, ainda, transitar pelo mundo formal axiomático.

Respostas incorretas:

Oito licenciandos erraram a resposta a esse item. A4 e A13 apenas escreveram as respostas: A4 indicou que "a sequência tende a zero", sem qualquer cálculo ou justificativa. A13 escreveu: "Sim, a figura converge para zero". A7, A9, A11 e A14 indicaram a mesma resposta, só que com símbolos: A7, A11 e A14 escreveram: " $\lim _{n \rightarrow \infty} \frac{n}{n^{2}+1}=0^{w}$ e A9 ainda errou a indicação da sequência, escrevendo apenas: " $\lim _{n \rightarrow \infty} n=0^{"}$; não houve, portanto, justificativa para a resposta. A12 escreveu: " $\lim _{n \rightarrow \infty} \frac{n}{n^{2}+1}=\frac{\infty}{\infty^{2}+1}=1$ " e A15 indicou que " $\lim _{n \rightarrow \infty} \frac{n}{n^{2}+1}=0^{m}$ e depois listou as substituições de $\mathrm{n}$ por 1, 10, 100 e 1.000, com os respectivos cálculos do limite, para concluir: "O limite pela direita se aproxima de zero".

Ainda surgem características do mundo corporificado, como na resposta de A15, que precisou substituir $\mathrm{n}$ por vários valores numéricos para dar a resposta; os demais alunos que erraram parecem entender que devem operar com os símbolos, ou seja, transitar pelo mundo operacional simbólico, mas não sabem desenvolver os procedimentos.

Numa síntese das respostas ao item $a$, considera-se que a maior parte dos participantes mostram não saber expressar o conceito de sequência convergente em linguagem simbólica, haja vista que nenhum deles indicou a definição formal, com uso de $\varepsilon$. Reconhecem a convergência na figura, até conseguem visualizar o ponto de convergência, mas permanecem no estágio da matemática prática.

Já no item $b$, as tentativas de usar símbolos para calcular o limite da sequência esbarram em dificuldades com a linguagem matemática; assim, parece-nos que os alunos ainda estão no estágio da matemática prática, pois não houve qualquer "demonstração formal" para justificar a resposta sobre o limite da sequência.

Nas respostas a ambos os itens, também é possível apontar “já-encontrados” que influenciam positiva ou negativamente as justificativas dos alunos. Na resposta ao item $a$, por exemplo, consideramos que A4, ao escrever "Não, pois podemos verificar a convergência da esquerda para a direita, contudo, da direita para a esquerda não é possível", usou um conhecimento inerente ao assunto "limite", mas pode ter pensado na representação geométrica de limite de uma função em um ponto, como a da função dada por $f(x)=\frac{1}{x-2}$, indicada na figura 2 : 


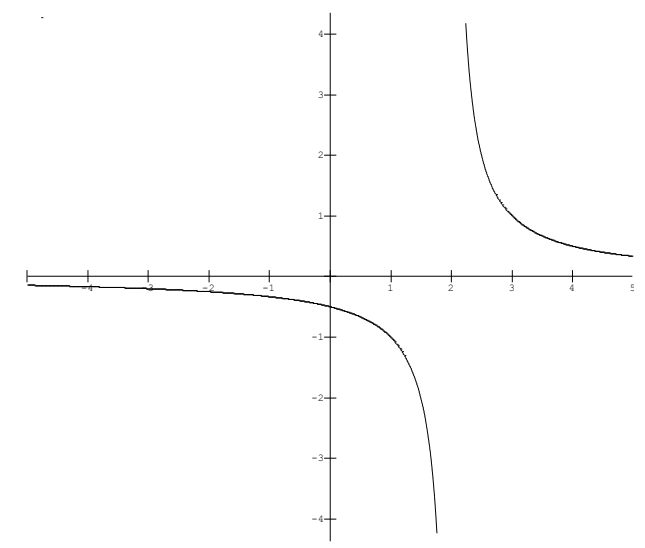

Figura 2 - Representação gráfica da função $f(x)=\frac{1}{x-2}$

Fonte: elaboração dos autores

Esse "já-encontrado" pode ter influenciado o respondente quando visualizou o gráfico da sequência e pensou em termos de limite à esquerda e à direita.

Já A2, na resposta ao item $b$, pode ter usado um conhecimento anterior, de que o valor de uma fração com numerador e denominador positivos e denominador maior que o numerador está entre 0 e 1; assim, justificou corretamente a convergência da sequência, apesar de não saber usar representações simbólicas para o cálculo do limite.

Outra dificuldade que pode influenciar negativamente a aprendizagem dos futuros alunos desses licenciandos é relacionada ao sinal de igualdade. Ponte, Branco e Matos (2009) consideram que há pelo menos três significados para o sinal de igualdade, relacionados à noção operacional, à ideia de equivalência e à noção de relação.

O primeiro significado é o mais usado nos anos iniciais, como, por exemplo, quando "os alunos realizam operações de um modo sequencial, da esquerda para a direita, usando o sinal de igual tanto como 'separador' entre dois raciocínios como para introduzir um novo resultado" (Ponte, Branco \& Matos, 2009, p. 22).

Trivilin e Ribeiro (2015) consideram que, em listas de exercícios do tipo "3+4=", os alunos "aprendem que devem armar e efetuar a operação e, na sequência, dar a resposta após o sinal de igualdade.” (p. 45, grifo dos autores).

Outro significado, ligado à ideia de equivalência entre expressões, é o que aparece em equações, como, por exemplo, $5+x=13$, em que se pergunta qual o valor de $x$ que torna equivalentes as expressões nos dois membros. Finalmente, como sinal indicativo da noção de relação de dependência entre duas variáveis, aparece em equações do tipo $\mathrm{y}=\mathrm{x}+7$.

Kieran (1981) comenta que, frequentemente, os alunos usam o sinal de igual apenas como um "link" entre diversos passos e exemplifica um erro realizado por estudantes de curso superior, ao calcular a derivada de uma função: os alunos encadearam o sinal de igualdade desde a expressão " $\mathrm{y}=\mathrm{f}(\mathrm{x})$ " até o resultado final da derivada da função $\mathrm{f}$, desenvolvendo os passos da derivação e ligando-os com o sinal de igual. 
Também analisando significados atribuídos à igualdade, Badaró e Lima (2011) trazem sua interpretação conforme a teoria dos Três Mundos da Matemática. Assim, no mundo corporificado, "quando o sinal de igualdade é encontrado, ele é relacionado a coisas concretas, como indicar o resultado de uma contagem ou o lugar onde a resposta de uma expressão deve ser colocada" (p. 3).

Já no mundo simbólico, quando Gray e Tall (1994) se referem à forma como processo e conceito são combinados em um "proceito" e exemplificam com a notação " $\lim _{n \rightarrow \infty} a_{n}$ ", parecem estar indicando que os alunos entendem a notação como o próprio processo e talvez por esse motivo tendem a encadear passos, como no exemplo dado por Kieran (1981), citado acima, e como nos erros cometidos pelos alunos A3, A5 e A8, ao responderem ao item $b$ da questão proposta nesta pesquisa.

No mundo formal, segundo Badaró e Lima (2011, p. 4), o símbolo de igualdade "extrapola o entendimento que lhe é dado no mundo simbólico, passando a ser entendido como a indicação de uma verdade, resultado de uma prova, ou então, algo que ainda precisa ser provado para alcançar essa situação". Esse entendimento da igualdade parece não ter sido atingido pelos participantes desta pesquisa, porque a ênfase das respostas ao item $b$ foi no cálculo, no encadeamento das expressões encontradas. Suas dificuldades, simbólicas e formais, podem ter feito com que nenhum dos 15 alunos respondesse corretamente a esse item da questão.

\section{CONSIDERAÇÕES FINAIS}

A pesquisa aqui relatada faz parte de uma investigação maior, apoiada pelo Edital Universal do $\mathrm{CNPq}$, que, além da análise de erros cometidos por licenciandos em questões sobre sequências, propõe-se a desenvolver atividades sobre sequências numéricas para serem aplicadas em aulas de Cálculo ou Análise dos cursos de Licenciatura em Matemática das instituições envolvidas na pesquisa.

Pela análise que foi feita sobre as respostas à questão apresentada neste artigo, concluímos que esses 15 licenciandos estão ainda no estágio da matemática prática, pois precisam apoiar-se em elementos corporificados para justificar suas respostas; além disso, parecem, também, ter dificuldades relacionadas à linguagem matemática que, talvez, não tenha sido suficientemente trabalhada em suas aulas ou, ainda, que o sinal de igualdade e o símbolo de limite, em seus significados como operadores, podem ter influenciado negativamente as respostas.

Ball, Thames e Phelps (2008, p. 395), com base nas ideias de Shulman, propuseram uma categorização para o conhecimento matemático para o ensino, entendido como "o conhecimento matemático necessário para levar adiante o trabalho de ensinar matemática". Esses autores consideram, inicialmente, que muitos estudos questionam o que os professores precisam saber, quais conteúdos devem compreender. Mas, por lhes parecer óbvio que os professores precisam saber conteúdos e procedimentos que vão lecionar, decidiram 
determinar "que mais os professores precisam saber sobre matemática e como e onde os professores podem usar tal conhecimento matemático na prática" (Ibid., p. 395).

Pelos resultados desta pesquisa, parece que esses futuros professores estão ainda ancorados no mundo corporificado, apresentando alguns elementos do mundo simbólico, mas com dificuldades que precisam ser superadas antes de trabalhar com estudantes da educação básica. Em cursos de formação de professores, consideramos que é necessário fazer uma "jornada" pelos Três Mundos da Matemática, em relação a qualquer dos conceitos e procedimentos apresentados, iniciando com atividades de matemática prática, apoiadas em figuras e símbolos já conhecidos, para, aos poucos, atingir o estágio da matemática formal.

Espera-se que o futuro professor tenha conhecimento do formalismo envolvido nos conceitos e demonstrações sobre sequência, mas, como apontam Fiorentini e Oliveira (2013, p. 925), "o professor precisa saber que uma demonstração não deve ser, necessariamente, sempre formal e fazer parte de um sistema axiomático". Entretanto, para que tenha consciência dessa possibilidade, esse futuro professor precisa ter, durante seu curso, oportunidade de compreender os significados dos conceitos em cada um dos mundos.

Em um curso de formação inicial de professores, acreditamos que os docentes formadores podem proporcionar atividades que levem em conta não só o conhecimento formal, mas também os melhores exemplos e a melhor abordagem metodológica para que os futuros professores possam justificar afirmativas, verbais ou escritas, que serão apresentadas aos alunos.

No tocante às sequências numéricas, por exemplo, o futuro professor pode passar pelo mundo corporificado, trabalhando com padrões e generalidades, para mais adiante entender o simbolismo envolvido nas definições e propriedades e, finalmente, atingir o mundo formal, trabalhado, por exemplo, em disciplinas de Análise Matemática. Ao preparar suas aulas sobre sequências, o professor vai precisar voltar aos três mundos e transitar entre eles, para ter consciência das suas especificidades. Essas oportunidades de ir e vir entre os Três Mundos da Matemática devem ser estimuladas pelos docentes formadores, em qualquer conteúdo trabalhado nas disciplinas.

Considera-se que as discussões sobre dificuldades de licenciandos em Matemática deveriam ser estimuladas, para que a formação inicial os capacite a apresentar os conteúdos da educação básica de uma forma que passe pelos Três Mundos da Matemática e que envolva metodologias variadas e exemplos adequados ao nível de ensino dos alunos da educação básica. Dessa forma, acreditamos ser possível, nos cursos de formação inicial de Matemática, proporcionar a base para uma prática profissional adequada à escola atual, com todas as suas especificidades e necessidades.

\section{Referências}

Attorps, I. (2006). Mathematics teachers'conceptions about equations. Tese de Doutorado em Educação. Faculty of Behavioural Sciences, University of Helsinki. 
DOI: http://dx.doi.org/10.20396/zet.v24i3.8648090

Badaró, J. N. \& Lima, R. N. (2011). Uma jornada pela história do símbolo de igualdade com os três mundos da matemática. Atas da XIII Conferência Interamericana de Educação Matemática. Recife, Brasil.

Ball, D. L., Thames, M. H. \& Phelps, G. (2008). Content knowledge for teaching: what makes it special? Journal of Teacher Education, 59 (5), 389-407.

Barichello, L. (2008). Análise de resoluções de problemas de cálculo diferencial em um ambiente de interação escrita. Dissertação de Mestrado em Educação Matemática. Rio Claro, Instituto de Geociências e Ciências Exatas, Universidade Estadual Paulista.

Borasi, R. (1996). Reconceiving mathematics Instruction: a Focus on Errors. Norwood, NJ: Ablex Publishing Corporation.

Brasil, Ministério da Educação, Conselho Nacional de Educação. (2015). Resolução n ${ }^{\circ}$ 2, de $1^{\circ}$ de julho de 2015. Define as Diretrizes Curriculares Nacionais para a formação inicial em nível superior (cursos de licenciatura, cursos de formação pedagógica para graduados e cursos de segunda licenciatura) e para a formação continuada. Retirado em 25 de novembro, 2016, de: http://den.prograd.ufsc.br/files/2016/07/2.7.DiretrizesLicenciatura2015_ResolucaoCNEC P2_2015.pdf

Cury, H. N. (2007). Análise de erros: o que podemos aprender com as respostas dos alunos. Belo Horizonte: Autêntica.

Ferreira, L. (2011). Uma proposta de ensino de geometria hiperbólica: construção do "plano de Poincaré" com o uso do software Geogebra. Dissertação de Mestrado em Educação para a Ciência e a Matemática. Maringá: Universidade Estadual de Maringá.

Fiorentini, D. \& Oliveira, A. T. de C. C. (2013). O lugar das matemáticas na Licenciatura em Matemática: que matemáticas e que práticas formativas? BOLEMA, 27 (47), 917-938.

Gray, E. M. \& Tall, D. (1994). Duality, ambiguity and flexibility: a proceptual view of simple arithmetic. The Journal for Research in Mathematics Education, 16 (2), 115-141.

Hardy, N. (2009). Students' perceptions of institutional practices: the case of limits of functions in college level Calculus courses. Educational Studies in Mathematics, 72 (3), 341-358.

Kieran, C. (1981). Concepts associated with the equality symbol. Educational Studies in Mathematics, (12), 317-326

Lima, E. L. (1999). Análise real. 4. ed. Rio de Janeiro: IMPA. v. 1.

Lima, R. N. (2007). Equações algébricas no ensino médio: uma jornada por diferentes mundos da matemática. Tese de Doutorado em Educação Matemática. São Paulo: Pontifícia Universidade de São Paulo.

Lima, R. N. \& Tall, D. (2008). Procedural embodiment and magic in linear equations. Educational Studies in Mathematics, (67), p. 3-18, 2008. 
DOI: http://dx.doi.org/10.20396/zet.v24i3.8648090

Mação, D. P. (2014). Uma proposta de ensino para o conceito de derivada. Dissertação de Mestrado em Educação Matemática. São Paulo: Universidade Anhanguera de São Paulo.

Malta, I., Pesco, S. \& Lopes, H. (2002). Cálculo a uma variável: uma introdução ao Cálculo. Rio de Janeiro: PUC-Rio; São Paulo: Loyola. v. 1.

Moreira, P. C. \& Ferreira, A. C. (2013). O lugar da Matemática na Licenciatura em Matemática. BOLEMA, 27 (47), 981-1005.

Nomura, J. I. (2014). Esquemas cognitivos e mente matemática inerentes ao objeto matemático autovalor e autovetor: traçando diferenciais na formação do engenheiro. Tese de Doutorado em Educação Matemática. São Paulo: Pontifícia Universidade Católica de São Paulo.

Ponte, J. P., Branco, N. \& Matos, A. (2009). Álgebra no ensino básico. Lisboa: Ministério da Educação.

Ramos, M. L. P. D. \& Curi, E. (2014). O uso do erro como estratégia didática: uma nova perspectiva na reconstrução do conhecimento. Perspectivas da Educação Matemática, Campo Grande, 7 (13), 84-102.

Santos, J. R. V., Buriasco, R. L. C. \& Ciani, A. B. (2008). Avaliação como prática de investigação e análise da produção escrita em matemática. Revista de Educação, (25), $35-45$.

Shulman, L. S. (1986). Those who understand: knowledge growth in teaching. Educational Researcher, 15 ( 2), 4-14.

Shulman, L. S. (1987). Knowledge and teaching: foundations of the New Reform. Harvard Educational Review, 57 (1), 1-22.

Stewart, J. (2001). Cálculo. São Paulo: Pioneira. v. 2.

Tall, D. (2004). Thinking through three worlds of mathematics. Proceedings of the 28th Meeting of the International Conference for the Psychology of Mathematics Education. Bergen, Norway. p. 281-288.

Tall, D. (2013). How Humans Learn to Think Mathematically: Exploring the Three Worlds of Mathematics. New York: Cambridge University Press.

Tall, D. \& Vinner, S. (1981). Concept image and concept definitionin mathematics with particular reference to limits and continuity. Educational Studies in Mathematics, (12), 151-169.

Trivilin, L. R. \& Ribeiro, A. J. (2015). Conhecimento matemático para o ensino de diferentes significados do sinal de igualdade: um estudo desenvolvido com professores dos anos iniciais do ensino fundamental. BOLEMA, 29 (51), 38-59. 
DOI: http://dx.doi.org/10.20396/zet.v24i3.8648090

Zubieta, G. \& Meza, R. (2008). Visualization in the teaching/learning of calculus. Proceedings of the 11th International Congress on Mathematical Education. Monterrey, Mexico. Retirado em 09 de março, 2016, de: http://tsg.icme11.org/tsg/show/21. 